\title{
Quantum statistics of overlapping modes in open resonators
}

\author{
Gregor Hackenbroich, Carlos Viviescas, and Fritz Haake \\ Fachbereich Physik, Universität Duisburg-Essen, 45117 Essen, Germany
}

(Dated: October 31, 2018)

\begin{abstract}
We study the quantum dynamics of optical fields in weakly confining resonators with overlapping modes. Employing a recently developed quantization scheme involving a discrete set of resonator modes and continua of external modes we derive Langevin equations and a master equation for the resonator modes. Langevin dynamics and the master equation are proved to be equivalent in the Markovian limit. Our open-resonator dynamics may be used as a starting point for a quantum theory of random lasers.
\end{abstract}

PACS numbers: 42.50.Ar, 42.55.Ah, 42.60.Da, 42.55.zz

\section{INTRODUCTION}

The interaction of a single resonator mode with an external optical field leads to damping and noise for the intracavity mode. The textbook example is a single oscillator linearly coupled to a continuum of harmonic oscillators 1, 2, 3, 4]. Less understood and in fact object of ongoing debate 5, 6, 7, 8, 9, 10, 11, 12, 13] are the damping and noise properties of multimode fields in resonators. Multimode fields show an excess noise or Petermann factor [5, 7, 14]. In laser systems this factor gives rise to a peculiar enhancement of the laser linewidth above the fundamental Schawlow-Townes value; this enhancement was measured in recent experiments on unstable laser cavities 15, 16]. Excess noise and the enhancement of the laser linewidth may be attributed to the nonorthogonality and the spectral overlap of the cavity eigenmodes 7] in the presence of the coupling to the external field.

While the phenomenon of excess noise has been known for more than 20 years, there is still no complete description of the quantum statistics of overlapping modes. A few example systems were discussed 8, 9] from a quantum mechanical point of view. Recently several authors 10, 11, 12, 13] proposed quantum Langevin and master equations for multimode fields; however, the status of these equations remained unclear as they were not derived from rigorously quantized electromagnetic fields. This is in contrast to the quantum properties of a singlemode field (linearly coupled to an external heat bath) which are known [1, 3, 4] for arbitrary damping strength and arbitrary heat-bath temperature.

The goal of the present paper is to derive and clarify the status of stochastic equations for the field dynamics in resonators with overlapping modes. The experimental motivation for our work derives both from realizations [15, 16] of unstable laser cavities and from recent experiments of highly disordered dielectrics which form mirrorless so-called random lasers 17, 18]. We address the field dynamics both within the Heisenberg picture (in terms of quantum Langevin equations) and within the Schrödinger picture (employing a master equation for the reduced density matrix of the cavity modes). We go beyond previous work in the following respects: (i) We derive the field dynamics starting from rigorously quan- tized electromagnetic fields. In particular, no restriction of the dimensionality and the vector character of the field strengths are indulged in. Keeping only resonant terms in the field Hamiltonian and adopting a Markov approximation for the memory of the "bath" (i.e., the electromagnetic field outside the resonator), we obtain the equations of motion for the intracavity field, including expressions for all damping and noise forces. (ii) Our derivation clarifies the status of the resulting stochastic equations. In particular, we show that they correctly describe separated as well as spectrally overlapping resonances. Corrections would only be important if the resonance widths were to become comparable to the resonance frequencies themselves, a regime not encountered in optical or even microwave resonators. (iii) We derive a representation of our master equation in terms of non-orthogonal modes and compare our result with master equations proposed earlier 10, 11, 13. This allows us to specify the physical conditions under which those previously proposed master equations hold.

\section{FIELD QUANTIZATION FOR OPEN-RESONATOR GEOMETRIES}

There is nothing to add to the familiar canonical quantization of the electromagnetic field 1, 2]. The field components may be expanded in terms of any complete set of basis functions. As a matter of convenience one usually employs eigenmodes pertinent to the given geometry and respecting the physical boundary conditions. Such expansions are at issue here.

In the presence of a more or less open resonator, one often wants to distinguish between "inside" and "outside" even though any opening, i.e., a hole in the material walls of the resonator gives to the latter concepts an element of arbitrariness. Any choice of a fictitious surface covering the hole yields its own inside/outside separation. Moreover, different boundary conditions may be imposed at the chosen separating surface. Nevertheless, each such surface and boundary condition entail eigenmodes allowing us to represent the electromagnetic field almost everywhere, with the qualifier "almost" reminding us of the fact that the expansion cannot be expected to converge 
pointwise everywhere, and in particular not on an arbitrarily chosen boundary.

The freedom in choosing the separating surface and the boundary condition thereon may be used 19, 20, 21] to define a discrete set of inside modes which vanish outside; and similarly a continuum (or even several continua distinguished by a "channel" index) of outside modes which vanish inside and fulfill scattering-type boundary conditions at infinity; a scattering condition specifies a single channel for a wave coming in from infinity and entails different amplitudes for the partial waves going out through the various channels. Canonical quantization then amounts to representing the coefficients of the mode expansion of the electromagnetic field by creation and annihilation operators $\left\{a_{\lambda}, a_{\lambda}^{\dagger}\right\}$ of photons of the $\lambda$ th inside mode and likewise $\left\{b_{m}(\omega), b_{m}^{\dagger}(\omega)\right\}$ of photons of the $(m, \omega)$ th outside mode, where $m$ labels channels (including polarization) and $\omega$ distinguishes modes within the channel continuum. The bosonic commutators read

$$
\begin{aligned}
{\left[a_{\lambda}, a_{\lambda^{\prime}}^{\dagger}\right] } & =\delta_{\lambda, \lambda^{\prime}}, \\
{\left[b_{m}(\omega), b_{m^{\prime}}^{\dagger}\left(\omega^{\prime}\right)\right] } & =\delta_{m m^{\prime}} \delta\left(\omega-\omega^{\prime}\right) ;
\end{aligned}
$$

moreover, inside operators commute with outside ones.

It was shown in two previous papers 20, 21] that the Hamiltonian of the electromagnetic field can be rigorously expressed as the following bilinear form in the foregoing creation and annihilation operators,

$$
\begin{gathered}
H=\sum_{\lambda} \hbar \omega_{\lambda} a_{\lambda}^{\dagger} a_{\lambda}+\sum_{m} \int d \omega \hbar \omega b_{m}^{\dagger}(\omega) b_{m}(\omega) \\
+\hbar \sum_{\lambda, m} \int d \omega\left[\mathcal{W}_{\lambda m}(\omega) a_{\lambda}^{\dagger} b_{m}(\omega)\right. \\
\left.+\mathcal{V}_{\lambda m}(\omega) a_{\lambda} b_{m}(\omega)+\text { H.c. }\right] .
\end{gathered}
$$

Here, the inside mode frequencies $\omega_{\lambda}$ as well as the insideoutside coupling amplitudes $\mathcal{W}_{\lambda m}, \mathcal{V}_{\lambda m}$ reflect the choices made for the separating surface. The latter amplitudes are integrals, taken over the separating surface, of products of inside (or outside) mode functions with derivatives of outside (inside) mode functions. Specifically, if the von-Neumann boundary condition

$$
\mathbf{n} \times\left.\left[\nabla \times \mathbf{u}_{\lambda}\right]\right|_{\partial I}=0
$$

was imposed for the inside modes $\mathbf{u}_{\lambda}$ and the Dirichlet boundary condition

$$
\mathbf{n} \times\left.\mathbf{v}_{m}(\omega)\right|_{\partial I}=0
$$

for the outside modes $\mathbf{v}_{m}(\omega)$ at the boundary $\partial I$ (with normal vector $\mathbf{n}$ ), the coupling amplitudes are given by

$$
\begin{aligned}
& \mathcal{W}_{\lambda m}(\omega)=\frac{c^{2}}{2 \sqrt{\omega_{\lambda} \omega}} \int_{\partial I} d^{2} r \mathbf{u}_{\lambda}^{*}(\mathbf{r}) \cdot\left\{\mathbf{n} \times\left[\nabla \times \mathbf{v}_{m}(\omega, \mathbf{r})\right]\right\},(5) \\
& \mathcal{V}_{\lambda m}(\omega)=\frac{c^{2}}{2 \sqrt{\omega_{\lambda} \omega}} \int_{\partial I} d^{2} r \mathbf{u}_{\lambda}(\mathbf{r}) \cdot\left\{\mathbf{n} \times\left[\nabla \times \mathbf{v}_{m}(\omega, \mathbf{r})\right]\right\} .(6)
\end{aligned}
$$

If the physical boundary conditions on material surfaces entail time reversal invariance, the amplitudes $\mathcal{W}, \mathcal{V}$ can be chosen real and become identical, $\mathcal{W}=\mathcal{V}$.

The derivation of the Hamiltonian takes full account of three spatial dimensions as well as of the vector character of the electric and magnetic fields $\mathbf{E}(\mathbf{x}, t)$ and $\mathbf{B}(\mathbf{x}, t)$; in brief, the Heisenberg equations of motion generated by that $H$ are equivalent to the quantized Maxwell equations. Moreover, by composing the inside (outside) mode functions with coefficients $\left\{a_{\lambda}, a_{\lambda}^{\dagger}\right\}\left(\left\{b_{m}(\omega), b_{m}^{\dagger}(\omega)\right\}\right)$ one gets the fields $\mathbf{E}(\mathbf{x}, t)$ and $\mathbf{B}(\mathbf{x}, t)$ inside (outside); truncated such compositions will be robust against small changes of the separating surface unless an unreasonable choice was made to begin with.

Hamiltonians of the above form have been used [1, 6] in quantum optics for high-quality resonators; there, inside excitations are but slowly dissipated to the outside, with life times long not only compared to typical internal periods $2 \pi / \omega_{\lambda}$ but even to beat periods $2 \pi / \Delta \omega$, where $\Delta \omega$ denotes the modulus of the typical frequency spacing of neighboring internal modes. In such applications the antiresonant terms $\mathcal{V}_{\lambda m} a_{\lambda} b_{m}+\mathcal{V}_{\lambda m}^{*} a_{\lambda}^{\dagger} b_{m}^{\dagger}$ can be neglected. However, the derivation of Refs. 20, 21] also secures validity of Hamiltonian (2) for very much open resonators where the outside field causes the inside resonances to overlap, the main case of interest in the present paper. The Hamiltonian (2) even remains valid in the extreme case of overdamped inside excitations, where mode frequencies $\omega_{\lambda}$ are overwhelmed by large escape rates $\kappa$; it is only in that extreme situation, which appears as not of interest in optics, that the antiresonant terms would be important.

\section{LANGEVIN EQUATIONS FOR OVERLAPPING RESONANCES}

While Hamiltonian (2) describes the coupled dynamics of the inside and outside fields, its principle application is to separate descriptions of those two subsystems. As already indicated above, the continua of outside modes tend to act as a "bath" damping the discrete inside modes. The effectively irreversible dynamics of the inside modes becomes manifest when the bath degrees of freedom are eliminated. The Heisenberg equations of motion of the inside amplitudes $a_{\lambda}, a_{\lambda}^{\dagger}$ then take the form of Langevin equations, in which the outside amplitudes $b_{m}(\omega), b_{m}^{\dagger}(\omega)$ enter only with their initial values in inhomogeneities, the Langevin noise forces. Due to the bilinear form of Hamiltonian (2) the Langevin equations can be determined rigorously by diagonalization of $H$, i.e., without resorting to perturbation expansions (for a single "system" oscillator the diagonalization was performed in, e.g., Refs. 22] and [4]).

The Langevin equations to be noted in the present section are simplified in three respects. First, we bar all antiresonant terms, assuming the absence of overdamping. Second, we confine the discussion to a Markovian situa- 
tion valid for times larger than "bath correlation times" $\tau_{\text {bath }}$ (like the thermal time $\tau_{\text {bath }}^{\text {th }}=\hbar / k_{B} T$ ); the existence of a time scale separation is thus assumed, such that all inside lifetimes are much in excess of $\tau_{\text {bath }}$. Third, to save space we do not bother to pedantically write out the so-called frequency-shift terms which are rarely needed in practice. The limit in question yields the Langevin equations 21]

$$
\dot{a}_{\lambda}(t)=-1 \omega_{\lambda} a_{\lambda}(t)-\sum_{\mu} \gamma_{\lambda \mu} a_{\mu}(t)+F_{\lambda}(t),
$$

wherein the damping matrix $\gamma_{\lambda \mu}$ and the noise force $F_{\lambda}$ are given in terms of the inside-outside coupling amplitudes $\mathcal{W}_{\lambda m}$ as

$$
\begin{aligned}
\gamma_{\lambda \mu} & =\pi\left(\mathcal{W} \mathcal{W}^{\dagger}\right)_{\lambda \mu}, \\
F_{\lambda}(t) & =-1 \int d \omega \mathrm{e}^{-1 \omega\left(t-t_{0}\right)} \sum_{m} \mathcal{W}_{\lambda m} b_{m}\left(\omega, t_{0}\right) .
\end{aligned}
$$

Note that the damping matrix $\gamma$ is non-negative and Hermitian; the neglected frequency-shift terms would amount to an anti-Hermitian addition to $\gamma$. Under conditions of time reversal invariance the matrix $\gamma$ is even real symmetric. The Markovian limit mentioned allows us to drop the frequency dependence of the coupling amplitudes $\mathcal{W}$ over the spectral range in consideration and to restrict the time span passed since the initial moment $t_{0}$ as $t-t_{0} \gg \tau_{\text {bath }}$. In that limit the force has a white spectrum according to $\left\langle F_{\lambda}(t)\right\rangle=0$, and the noise

$$
\begin{aligned}
\left\langle F_{\lambda}^{\dagger}(t) F_{\mu}\left(t^{\prime}\right)\right\rangle & =2 \gamma_{\mu \lambda} n_{\text {th }} \delta\left(t-t^{\prime}\right), \\
\left\langle F_{\lambda}(t) F_{\mu}^{\dagger}\left(t^{\prime}\right)\right\rangle & =2 \gamma_{\lambda \mu}\left(1+n_{\text {th }}\right) \delta\left(t-t^{\prime}\right) .
\end{aligned}
$$

The second two-time correlation functions follows from the first and the commutation relations (1b). The remaining second moments vanish, $\left\langle F_{\lambda}(t) F_{\mu}\left(t^{\prime}\right)\right\rangle=0=$ $\left\langle F_{\lambda}^{\dagger}(t) F_{\mu}^{\dagger}\left(t^{\prime}\right)\right\rangle$, and higher-order moments follow from the ones of orders 1 and 2 according to Gaussian statistics. The thermal number of photons $n_{\mathrm{th}}=[\exp (\hbar \bar{\omega} / k T)-$ $1]^{-1}$ appearing in the second moments must be taken as frequency independent throughout the spectral range of inside frequencies $\omega_{\lambda}$ under consideration. In the limit $k T \ll \hbar \bar{\omega}$ we recover the Langevin equations of Bardroff and Stenholm [10].

The appearance of a nondiagonal damping matrix $\gamma=\pi \mathcal{W} \mathcal{W}^{\dagger}$ signals that the Langevin equation may legitimately be applied to the case of overlapping resonances, in which typical matrix elements $\gamma_{\lambda \mu}$ are larger than a typical nearest-neighbor spacing $\Delta \omega$ of frequencies. However, all elements of $\gamma$ must be smaller in magnitude than the frequencies $\omega_{\lambda}$ themselves as antiresonant terms were dropped in the derivation of Eq. (7). Conversely, we could specialize to the weak-coupling regime $\left|\gamma_{\lambda \mu}\right| \ll \Delta \omega$. Then, lowest-order perturbation theory simply amounts to dropping the off-diagonal elements of the damping matrix, $\gamma_{\lambda \mu} \rightarrow 0$ for $\lambda \neq \mu$, whereupon the Langevin equation (7) simplifies so as to describe a set of mutually independent damped harmonic oscillators.
For some applications it will be helpful to rewrite the above Langevin equation with the non-Hermitian matrix

$$
\mathcal{H}_{\lambda \mu}=\hbar \omega_{\lambda} \delta_{\lambda \mu}-1 \hbar \gamma_{\lambda \mu}
$$

diagonalized. The eigenvalues of $\mathcal{H}$ will then represent "true" resonances of the cavity. As a "penalty" for that change of representation one would have to work with non-standard commutation relations for the operators connected with the eigenvectors of $\mathcal{H}$ (see Sec. VII).

A final remark on the dynamics of the outside field is in order here. The time scale separation assumed in deriving the above Langevin equations is effective outside as well as inside as the outside field evolves in adiabatic equilibrium with the inside field for times $t-t_{0} \gg \tau_{\text {bath }}$. For the quantitative treatment by the quantum optical input-output formalism or, equivalently, scattering theory we refer the interested reader to $[1,[2,21]$.

\section{MASTER EQUATION FOR OVERLAPPING RESONANCES}

We now come to the central section of the present paper. Switching to the Schrödinger picture we present the master equation for the reduced density operator $\rho(t)$ of the inside field equivalent to the Langevin equation of the preceding section,

$$
\begin{aligned}
\dot{\rho} & =-1 \sum_{\lambda} \omega_{\lambda}\left[a_{\lambda}^{\dagger} a_{\lambda}, \rho\right]+\left(1+n_{\mathrm{th}}\right) \sum_{\lambda \mu} \gamma_{\lambda \mu}\left\{\left[a_{\mu}, \rho a_{\lambda}^{\dagger}\right]\right. \\
& \left.+\left[a_{\mu} \rho, a_{\lambda}^{\dagger}\right]\right\}+n_{\mathrm{th}} \sum_{\lambda \mu} \gamma_{\lambda \mu}\left\{\left[a_{\lambda}^{\dagger}, \rho a_{\mu}\right]+\left[a_{\lambda}^{\dagger} \rho, a_{\mu}\right]\right\} .
\end{aligned}
$$

This equation generalizes the familiar quantum optical master equation for a single damped harmonic oscillator to many oscillators coupled by the (off-diagonal elements of the) damping matrix $\gamma_{\lambda \mu}$. The latter coupling is important when the damping is strong enough to cause spectral overlap of modes. The first double sum, proportional to $1+n_{\mathrm{th}}$, describes spontaneous and induced emission of photons towards the outside while the second double sum, proportional only to $n_{\mathrm{th}}$, describes absorption from the outside; that interpretation is easily checked by employing the Fock representation, i.e. the representation in terms of eigenstates of the photon number operators $a_{\lambda}^{\dagger} a_{\lambda}$.

Systematic and stochastic forces are not as clearly separated here as in the Langevin equation. In order to bring about such distinction here as well, and to prepare for the proof of equivalence of Eqs. (17) and (11) we may imagine the density operator $\rho(t)$ at time $t$ antinormally ordered in the annihilation and creation operators (all $a$ 's to the left of all $a^{\dagger}$ 's); further, we rearrange the commutators in the right-hand side of the master equation (11) such 
that $\dot{\rho}$ becomes anti-normally ordered provided $\rho(t)$ is,

$$
\begin{aligned}
\dot{\rho}= & -1 \sum_{\lambda} \omega_{\lambda}\left\{\left[a_{\lambda}, \rho a_{\lambda}^{\dagger}\right]-\left[a_{\lambda} \rho, a_{\lambda}^{\dagger}\right]\right\} \\
& +\sum_{\lambda \mu} \gamma_{\lambda \mu}\left\{\left[a_{\mu}, \rho a_{\lambda}^{\dagger}\right]+\left[a_{\mu} \rho, a_{\lambda}^{\dagger}\right]\right\} \\
& +2 n_{\mathrm{th}} \sum_{\lambda \mu} \gamma_{\lambda \mu}\left[\left[a_{\mu}, \rho\right], a_{\lambda}^{\dagger}\right] .
\end{aligned}
$$

The latter form of the master equation preserves antinormal ordering of $\rho(t)$ at all times. Moreover, we have now separated reversible drift terms (proportional to the frequencies $\left.\omega_{\lambda}\right)$, irreversible drift terms $\left(\propto \gamma_{\lambda \mu}\right)$ not involving the thermal number of quanta $n_{\mathrm{th}}$, and noise generated diffusion terms $\left(\propto n_{\mathrm{th}}\right)$; the latter interpretation will become obvious in the following section where a representation based on coherent states will be employed.

\section{EQUIVALENCE OF LANGEVIN AND MASTER EQUATIONS}

There are various ways of demonstrating the equivalence of the Langevin equations (7) and the master equation (11) and (12). A somewhat indirect (and, in fact, the most laborious) way would be to start from the Liouvillevon Neumann equation $\dot{\rho}_{\text {in } \oplus \text { out }}(t)=-1\left[H, \rho_{\text {in } \oplus \text { out }}(t)\right] / \hbar$ for the density operator of the full electromagnetic field and stick to the Schrödinger picture in eliminating the outside field, employing the same approximations as in deriving the Langevin equations. A standard, and more economical, procedure is to show that Eq. (7) and (12) entail the same evolution equations for all mean values $\left\langle\prod_{\lambda}\left(a_{\lambda}^{\dagger}\right)^{m_{\lambda}} \prod_{\mu}\left(a_{\mu}\right)^{n_{\mu}}\right\rangle(t)$ with integer exponents $\left\{m_{\lambda}, n_{\mu}\right\}$. We here prefer to employ a less familiar but particularly elegant method.

If we consistently stick to antinormal ordering of $\rho(t)$ we may write the commutators in the master equation (12) as differential operators as $[a,(\cdot)] \rightarrow\left(\partial / \partial a^{\dagger}\right)(\cdot)$ and $\left[(\cdot), a^{\dagger}\right] \rightarrow(\partial / \partial a)(\cdot)$. We may then just as well degrade all creation and annihilation operators to complex c-number variables and the density operator to a real function of those variables,

$$
a_{\lambda} \rightarrow \alpha_{\lambda}, \quad a_{\lambda}^{\dagger} \rightarrow \alpha_{\lambda}^{*}, \quad \rho(t) \rightarrow P\left(\left\{\alpha, \alpha^{*}\right\}, t\right) .
$$

The function $P$ in question is the familiar GlauberSudarshan quasiprobability [1] which has as its moments expectation values of normally ordered observables,

$$
\begin{aligned}
& \left\langle\prod_{\lambda}\left(a_{\lambda}^{\dagger}\right)^{m_{\lambda}} \prod_{\mu}\left(a_{\mu}\right)^{n_{\mu}}\right\rangle(t)= \\
& \int\left\{\prod_{\lambda} d \alpha_{\lambda} d \alpha_{\lambda}^{*}\left(\alpha_{\lambda}^{*}\right)^{m_{\lambda}}\left(\alpha_{\lambda}\right)^{n_{\lambda}}\right\} P\left(\left\{\alpha, \alpha^{*}\right\}, t\right) .
\end{aligned}
$$

The master equation (12) then becomes the FokkerPlanck equation (with the shorthand $\partial / \partial \alpha_{\lambda} \equiv \partial_{\lambda}$, $\left.\partial / \partial \alpha_{\lambda}^{*} \equiv \partial_{\lambda}^{*}\right)$

$$
\begin{aligned}
\dot{P}=[ & -1 \sum_{\lambda} \omega_{\lambda}\left(\partial_{\lambda}^{*} \alpha_{\lambda}^{*}-\partial_{\lambda} \alpha_{\lambda}\right) \\
& \left.+\sum_{\lambda \mu} \gamma_{\lambda \mu}\left(\partial_{\lambda}^{*} \alpha_{\mu}^{*}+\partial_{\mu} \alpha_{\lambda}+2 n_{\mathrm{th}} \partial_{\mu} \partial_{\lambda}^{*}\right)\right] P .
\end{aligned}
$$

At this point the interpretation of the various terms in the master equation (12) given earlier becomes obvious.

In the same vein we may degrade the Langevin equation to the c-number equation

$$
\dot{\alpha}_{\lambda}(t)=-\frac{1}{\hbar} \sum_{\mu} \mathcal{H}_{\lambda \mu} \alpha_{\mu}(t)+\varphi_{\lambda}(t)
$$

where $\varphi_{\lambda}(t)$ is the c-number representative of the operator valued random force $F_{\lambda}(t)$. Gaussian statistics and zero mean are imparted to $\varphi(t)$. Moreover, since observables are consistently to be taken as normally ordered we must identify second moments as

$$
\left\langle\varphi_{\lambda}^{*}(t) \varphi_{\mu}\left(t^{\prime}\right)\right\rangle=\left\langle F_{\lambda}^{\dagger}(t) F_{\mu}\left(t^{\prime}\right)\right\rangle=2 n_{\mathrm{th}} \gamma_{\mu \lambda} \delta\left(t-t^{\prime}\right) ;
$$

while holding on to $\left\langle\varphi_{\lambda}(t)\right\rangle=0,\left\langle\varphi_{\lambda}(t) \varphi_{\mu}\left(t^{\prime}\right)\right\rangle=0$ and Gaussian factorization for the higher-order moments.

At this point the equivalence proof can be carried out by deducing the Fokker-Planck equation (15) from the c-number Langevin equation (16), and to that task we now turn. In order to make the argument as transparent as possible we drop mode indices; the initial time at which statistical independence of the inside and outside fields is assumed is taken as $t_{0}=0$. We start by considering a fixed realization of the time-dependent "external forces" $\varphi(t), \varphi^{*}(t)$. The solution of the Langevin equation is then a functional of the temporal course taken by the external force between the initial and the current time. Correspondingly, we introduce a density $W\left(\alpha, \alpha^{*}, t \mid\left\{\varphi(t), \varphi^{*}(t)\right\}\right)$ functionally depending on the realization of the external field under consideration; at the initial time $t=0$ the new density coincides with the Glauber-Sudarshan $P$-function, $W(0)=P(0)$. The density $W$ obeys the evolution equation

$$
\begin{aligned}
\dot{W}(t) & =\left(L_{0}+l(t)\right) W(t), \\
L_{0} & =-1 \omega\left(\partial^{*} \alpha^{*}-\partial \alpha\right)+\gamma\left(\partial^{*} \alpha^{*}+\partial \alpha\right), \\
l(t) & =-\left(\partial^{*} \varphi^{*}(t)+\partial \varphi(t)\right) .
\end{aligned}
$$

Clearly, we encounter a first-order partial differential equation since the random character of the external force is not yet invoked. The time independent part $L_{0}$ of the generator accounts for oscillation and damping, while $l(t)$ includes the time dependent forces $\varphi(t), \varphi^{*}(t)$. The formal solution reads $W(t)=\left(\exp \int_{0}^{t} d s\left(L_{0}+l(s)\right)\right)_{+} W(0)$, where $(\cdot)_{+}$denotes positive time ordering. Temporarily employing an "interaction picture" we split off the mo- 
tion generated by $L_{0}$ as

$$
\begin{aligned}
W(t) & =\mathrm{e}^{L_{0} t} \tilde{W}(t) \\
\tilde{W}(t) & =\left[\exp \int_{0}^{t} d s \tilde{l}(s)\right]_{+} P(0) \\
\tilde{l}(t) & =\mathrm{e}^{-L_{0} t} l(t) \mathrm{e}^{L_{0} t}=-\left[\varphi^{*}(t) \tilde{\partial}^{*}(t)+\varphi(t) \tilde{\partial}(t)\right] .
\end{aligned}
$$

Now we may take the average over the Gaussian ensemble of realizations of $\varphi(t), \varphi^{*}(t)$. The average of the $\varphi$ dependent density $W(t)$ is just the Glauber-Sudarshan $P$-function, $\left\langle W\left(\alpha, \alpha^{*}, t \mid\left\{\varphi(t), \varphi^{*}(t)\right\}\right)\right\rangle=P\left(\alpha, \alpha^{*}, t\right)$. Invoking the above first and second moments we get

$$
\begin{aligned}
\tilde{P}(t) & =\left\langle\left(\exp \left[\int_{0}^{t} d s \tilde{l}(s)\right]\right)_{+}\right\rangle P(0) \\
& =\left(\exp \left[\int_{0}^{t} d s \int_{0}^{t} d s^{\prime}\left\langle\varphi^{*}(s) \varphi\left(s^{\prime}\right)\right\rangle \tilde{\partial}^{*}(s) \tilde{\partial}\left(s^{\prime}\right)\right]\right)_{+} P(0) \\
& =\left(\exp \left[2 n_{\mathrm{th}} \gamma \int_{0}^{t} d s \tilde{\partial}^{*}(s) \tilde{\partial}(s)\right]\right)_{+} P(0)
\end{aligned}
$$

Upon differentiating with respect to time we have $\dot{\tilde{P}}(t)=$ $2 n_{\mathrm{th}} \gamma \tilde{\partial}^{*}(t) \tilde{\partial}(t) \tilde{P}(t)$, and going back to $P(t)=\mathrm{e}^{L_{0} t} \tilde{P}(t)$, we arrive at the Fokker-Planck equation (15) and have thus arrived at our goal.

We should add that a mathematically more satisfactory variant of the foregoing considerations would result from employing the so-called Ito calculus [1].

\section{STATIONARY SOLUTION OF THE MASTER EQUATION}

The general time-dependent solution of the FokkerPlanck equation (12) is, due to the linearity of the drift coefficients and the constancy of the diffusion matrix, easy to construct [23]. We are in fact facing a stochastic process of the Ornstein-Uhlenbeck type. The Gaussian distribution of the noise together with the linear evolution equation (16) imply that the stochastic variables must be Gaussian distributed. In particular, the stationary $P$ function is immediately checked to be

$$
\bar{P}\left(\left\{\alpha, \alpha^{*}\right\}\right)=\prod_{\lambda} \frac{1}{\pi n_{\mathrm{th}}} \exp \left(-\alpha_{\lambda}^{*} \alpha_{\lambda} / n_{\mathrm{th}}\right) .
$$

The dissipative coupling of the system modes is no longer visible in the stationary state; rather, we encounter the thermal equilibrium state one would also find in the absence of spectral overlap.

\section{STATUS OF LANGEVIN AND MASTER EQUATIONS AND RELATED LITERATURE}

Multimode fields are more complex than single-mode fields due the additional frequency scale set by the mean frequency spacing $\Delta \omega$ of internal modes. For resonators with overlapping modes the mode spectral broadening is comparable to $\Delta \omega$. Langevin or master equations may then only be used if they provide a nonperturbative description of damping and noise (in the sense that the mode decay rates $\kappa$ may exceed the mode spacing $\Delta \omega$ ).

To show that the Langevin (7) and the master equations (11) and (12) are non-perturbative in the abovementioned sense, we now drop the rotating-wave and Markov approximation and address the exact field dynamics. From the field Hamiltonian (2) one obtains the following set of Langevin equations

$$
\begin{aligned}
\dot{a}_{\lambda}(t)= & -1 \omega_{\lambda} a_{\lambda}(t)-\sum_{\mu} \int_{t_{0}}^{t} d t^{\prime}\left[\Gamma_{\lambda \mu}\left(t-t^{\prime}\right) a_{\mu}\left(t^{\prime}\right)\right. \\
& \left.+\Sigma_{\lambda \mu}\left(t-t^{\prime}\right) a_{\mu}^{\dagger}\left(t^{\prime}\right)\right]+f_{\lambda}(t)
\end{aligned}
$$

Non-Markovian effects and corrections to isolated-mode behavior are encoded in the autocorrelation functions

$$
\begin{gathered}
\Gamma_{\lambda \mu}\left(t-t^{\prime}\right)=\sum_{m} \int_{\omega_{m}}^{\infty} d \omega\left[\mathrm{e}^{-1 \omega\left(t-t^{\prime}\right)} W_{\lambda m}(\omega) W_{\mu m}^{*}(\omega)\right. \\
\left.-\mathrm{e}^{+1 \omega\left(t-t^{\prime}\right)} V_{\lambda m}^{*}(\omega) V_{\mu m}(\omega)\right] \\
\Sigma_{\lambda \mu}\left(t-t^{\prime}\right)=\sum_{m} \int_{\omega_{m}}^{\infty} d \omega\left[\mathrm{e}^{-1 \omega\left(t-t^{\prime}\right)} W_{\lambda m}(\omega) V_{\mu m}^{*}(\omega)\right. \\
\left.-\mathrm{e}^{+1 \omega\left(t-t^{\prime}\right)} V_{\lambda m}^{*}(\omega) W_{\mu m}^{*}(\omega)\right]
\end{gathered}
$$

The noise term takes the form

$$
\begin{gathered}
f_{\lambda}(t)=-1 \sum_{m} \int_{\omega_{m}}^{\infty} d \omega\left[\mathrm{e}^{-i \omega\left(t-t_{0}\right)} W_{\lambda m}(\omega) b_{m}\left(\omega, t_{0}\right)\right. \\
\left.+\mathrm{e}^{+1 \omega\left(t-t_{0}\right)} V_{\lambda m}^{*}(\omega) b_{m}^{\dagger}\left(\omega, t_{0}\right)\right] .
\end{gathered}
$$

Due to the bilinear form of the field Hamiltonian the exact Langevin equations are linear in the system and bath operators. In principle, their solution can be found by Laplace transformation. The details of the exact solution will depend both on the spectral strength of the bath and the frequency dependent coupling amplitudes $W_{\lambda m}(\omega)$ and $V_{\lambda m}(\omega)$.

The result (22)-25) holds for arbitrary damping. Here, our main focus is on quantum optical systems where the oscillation frequencies $\bar{\omega}$ much exceed the typical damping rates $\kappa$. Then our result can be used to compute corrections to the Markovian dynamics of Secs. III We substitute the ansatz $a_{\lambda}(t) \sim \exp (-1[\bar{\omega}-i \kappa] t)$ with the damping rate $\kappa$ into Eq. (22); here $\bar{\omega}$ includes a frequency shift $\delta \bar{\omega}$. One obtains four terms proportional to $a_{\mu}$ or $a_{\mu}^{\dagger}$, out of which three terms are strongly oscillating: the contribution $\propto V^{*} W\left(W V^{*}\right)$ has the oscillatory integrand $\sim \mathrm{e}^{-2 \mathrm{\imath} \bar{\omega} t^{\prime}}\left(\mathrm{e}^{+2 \mathrm{\imath} \bar{\omega} t^{\prime}}\right)$, respectively, and the term $\propto V^{*} W$ oscillates $\sim \mathrm{e}^{+21 \bar{\omega} t}$ with respect to $a_{\lambda}(t)$. To leading order in the ratio $|\delta \Omega| / \bar{\omega} \equiv|\delta \bar{\omega}-1 \kappa| / \bar{\omega}$ only the term $\propto W W^{*}$ must be kept. The corrections of higher order in $|\delta \Omega| / \bar{\omega}$ are very small for the systems of interest 
in quantum optics. We note that there are no corrections of order $|\delta \Omega| / \Delta \omega$; thus our field dynamics correctly describes the regime of overlapping modes.

So far we described the field dynamics in terms of eigenmodes of a conveniently chosen closed system. The associated mode operators $\left\{a_{\lambda}, a_{\lambda}^{\dagger}\right\}$ obey canonical commutation relations. The master equation (11) may equivalently be expressed in terms of a biorthogonal set of modes representing the resonances of the open cavity. It turns out that the operators associated with the resonances obey nonstandard commutation rules. We focus on the case $k T \ll \hbar \bar{\omega}$ and write Eq. (11) in the form

$$
\begin{aligned}
\dot{\rho} & =-\frac{1}{\hbar}\left(H_{\mathrm{eff}} \rho-\rho H_{\mathrm{eff}}^{\dagger}\right)+2 \sum_{\lambda \mu} \gamma_{\lambda \mu} a_{\mu} \rho a_{\lambda}^{\dagger}, \\
H_{\mathrm{eff}} & =\sum_{\lambda \mu} \mathcal{H}_{\lambda \mu} a_{\lambda}^{\dagger} a_{\mu} .
\end{aligned}
$$

The non-Hermitian matrix $\mathcal{H}$ was introduced in Eq. (10). The complex eigenvalues of $\mathcal{H}$ represent the resonances of cavity in the presence of the coupling to the bath. Generally, the eigenvalues are nondegenerate, and $\mathcal{H}$ can be diagonalized by a similarity transformation $\mathcal{H}=T \Omega T^{-1}$. The diagonal matrix $\Omega$ comprises the eigenvalues of $\mathcal{H}$ while $T$ is a general matrix with complex entries. In terms of two novel sets of operators

$$
\begin{aligned}
d_{n} & =\sum_{\lambda} T_{n \lambda}^{-1} a_{\lambda}, \\
e_{n}^{\dagger} & =\sum_{\lambda} a_{\lambda}^{\dagger} T_{\lambda n},
\end{aligned}
$$

the effective Hamiltonian takes the diagonal form

$$
H_{\mathrm{eff}}=\sum_{n} \Omega_{n} e_{n}^{\dagger} d_{n}
$$

This result resembles the diagonal field Hamiltonian of closed resonators. However, the peculiar properties of the open resonator dynamics are encoded in nonstandard commutation relations of the new operators: From Eqs. (28) and (29) and the canonical commutation rules for the operators $\left\{a_{\lambda}, a_{\lambda}^{\dagger}\right\}$ one finds, e.g., $\left[d_{m}, e_{n}^{\dagger}\right]=\delta_{m, n}$ and $\left[e_{m}, e_{n}^{\dagger}\right]=A_{m n}$ with $A \equiv T^{\dagger} T$. Substitution of Eq. (28) into Eq. (26) yields the master equation

$$
\dot{\rho}=-\frac{1}{\hbar}\left(H_{\mathrm{eff}} \rho-\rho H_{\mathrm{eff}}^{\dagger}\right)+\frac{1}{\hbar} \sum_{n m} A_{n m}\left(\Omega_{m}-\Omega_{n}^{*}\right) d_{m} \rho d_{n}^{\dagger}
$$

This is the master equation proposed by Lamprecht and Ritsch [13]. Our derivation provides a microscopic basis for that equation [24] and proves the equivalence to the master equation (111) in the limit $k T \ll \hbar \bar{\omega}$.

\section{Acknowledgments}

We thank H.-J. Sommers and D. V. Savin for helpful discussions. This work has been supported by the SFB/TR 12 der Deutschen Forschungsgemeinschaft.
[1] C. W. Gardiner and P. Zoller, Quantum Noise, 2nd edition (Springer, Berlin, 2000).

[2] D. F. Walls and G. J. Milburn, Quantum Optics (Springer, Berlin, 1994).

[3] U. Weiss, Quantum Dissipative Systems, 2nd edition (World Scientific, Singapore, 1999).

[4] F. Haake and R. Reibold, Phys. Rev. A 32, 2462 (1985).

[5] K. Petermann, IEEE J. Quantum Electron. 15, 566 (1979).

[6] S. M. Barnett and P. M. Radmore, Opt. Commun. 68, 364 (1988).

[7] A. E. Siegman, Phys. Rev. A 39, 1253 (1989); Phys. Rev. A 39, 1264 (1989).

[8] Ph. Goldberg, P. W. Milonni, and B. Sundaram, Phys. Rev. A 44, 1969 (1991).

[9] Ph. Grangier and J.-Ph. Poizat, Eur. Phys. J. D 1, 97 (1998).

[10] P. J. Bardroff and S. Stenholm, Phys. Rev. A 60, 2529 (1999); 61, 023806 (2000); 62, 023814 (2000).

[11] C. Lamprecht and H. Ritsch, Phys. Rev. Lett. 82, 3787 (1999).

[12] A. M. van der Lee, N. J. van Druten, M. P. van Exter, J. P. Woerdman, J. P. Poizat, and P. Grangier, Phys. Rev. Lett. 85, 4711 (2000).

[13] C. Lamprecht and H. Ritsch, Phys. Rev. A 65, 023803
(2002); 66, 053808 (2002).

[14] C. W. J. Beenakker, Phys. Rev. Lett. 81, 1829 (1998).

[15] Y.-J. Cheng, C. G. Fanning, and A. E. Siegman, Phys. Rev. Lett. 77, 627 (1996).

[16] M. A. van Eijkelenborg, A. M. Lindberg, M. S. Thijssen, and J. P. Woerdman, Phys. Rev. A 55, 4556 (1997).

[17] S. V. Frolov, Z. V. Vardeny, and K. Yoshino, Phys. Rev. B 57, 9141 (1998).

[18] H. Cao et al., Phys. Rev. Lett. 82, 2278 (1999); H. Cao et al., Phys. Rev. Lett. 84, 5584 (2000); H. Cao et al., Phys. Rev. Lett. 86, 4524 (2001).

[19] D. V. Savin, V. V. Sokolov, and H.-J. Sommers, Phys. Rev. E 67, 026215 (2003).

[20] G. Hackenbroich, C. Viviescas, and F. Haake, Phys. Rev. Lett. 89, 083902 (2002).

[21] C. Viviescas and G. Hackenbroich, Phys. Rev. A 67, 013805 (2003).

[22] P. Ullersma, Physica (Utrecht), 32, 27 (1966); 32, 56 (1966); 32, 74 (1966); 32, 90 (1966).

[23] H. Risken, The Fokker-Planck Equation, 2nd edition (Springer, Berlin, 1989).

[24] Our derivation shows that the master equation proposed in Ref. 11] may describe the field dynamics only in the case of complete mode degeneracy. 